\title{
Treatment outcome of localized prostate cancer by 70 Gy hypofractionated intensity-modulated radiotherapy with a customized rectal balloon
}

\author{
Hyunjung Kim, MD', Jun Won Kim, MD', Sung Joon Hong, MD², Koon Ho Rha, MD², \\ Chang-Geol Lee, MD', Seung Choul Yang, MD², Young Deuk Choi, MD², \\ Chang-Ok Suh, MD'1, Jaeho Cho, MD¹ \\ Departments of ${ }^{1}$ Radiation Oncology and ${ }^{2}$ Urology, Yonsei Cancer Center, Yonsei University College of Medicine, Seoul, Korea
}

Purpose: We aimed to analyze the treatment outcome and long-term toxicity of 70 Gy hypofractionated intensity-modulated radiotherapy (IMRT) for localized prostate cancer using a customized rectal balloon.

Materials and Methods: We reviewed medical records of 86 prostate cancer patients who received curative radiotherapy between January 2004 and December 2011 at our institution. Patients were designated as low (12.8\%), intermediate (20.9\%), or high risk (66.3\%). Thirty patients received a total dose of $70 \mathrm{~Gy}$ in 28 fractions over 5 weeks via IMRT (the Hypo-IMRT group); 56 received 70.2 Gy in 39 fractions over 7 weeks via 3-dimensional conformal radiotherapy (the CF-3DRT group, which served as a reference for comparison). A customized rectal balloon was placed in Hypo-IMRT group throughout the entire radiotherapy course. Androgen deprivation therapy was administered to 47 patients (Hypo-IMRT group, 17; CF-3DRT group, 30). Late genitourinary (GU) and gastrointestinal (GI) toxicity were evaluated according to the Radiation Therapy Oncology Group criteria.

Results: The median follow-up period was 74.4 months (range, 18.8 to 125.9 months). The 5-year actuarial biochemical relapsefree survival rates for low-, intermediate-, and high-risk patients were 100\%, 100\%, and $88.5 \%$, respectively, for the Hypo-IMRT group and $80 \%, 77.8 \%$, and $63.6 \%$, respectively, for the CF-3DRT group ( $p<0.046)$. No patient presented with acute or late GU toxicity $\geq$ grade 3 . Late grade $3 \mathrm{GI}$ toxicity occurred in 2 patients (3.6\%) in the CF-3DRT group and 1 patient (3.3\%) in the HypoIMRT group.

Conclusion: Hypo-IMRT with a customized rectal balloon resulted in excellent biochemical control rates with minimal toxicity in localized prostate cancer patients.

Keywords: Prostatic neoplasm, Hypofractionation, Rectal Balloon, Radiotherapy, Intensity modulation

\section{Introduction}

The optimum radiation treatment schedule for localized prostate cancer remains unknown. Although recent doseescalation studies showed improved biochemical and local control rates [1-3], prolonging the radiation schedule increases the financial burdens and inconveniences for patients. However, studies that determines prostate cancer sensitivity to fractionation in radiotherapy treatments point to an $\alpha / \beta$ value around $2.7 \mathrm{~Gy}[4,5]$, which is lower than that required for late

Received 2 May 2014, Revised 3 August 2014, Accepted 1 September 2014.

Correspondence: Jaeho Cho, MD, Department of Radiation Oncology, Yonsei University College of Medicine, 50 Yonsei-ro, Seodaemun-gu, Seoul 120-749, Korea. Tel: +82-2-2228-8095, Fax: +82-2-312-9033, E-mail: jjhmd@yuhs.ac

(c) This is an Open Access article distributed under the terms of the Creative Commons Attribution Non-Commercial License (http://creativecommons.org/ licenses/by-nc/3.0/) which permits unrestricted non-commercial use, distribution, and reproduction in any medium, provided the original work is properly cited.

www.e-roj.org 
rectal complications (5.4 $\pm 1.5 \mathrm{~Gy}$ ) [6]. Thus, hypofractionation is an attractive option for treatment of localized prostate cancer.

Because of normal tissue tolerance, escalation of the total dose and fractional dose requires precise radiation delivery techniques, such as intensity-modulated radiotherapy (IMRT) and strict immobilization devices. Rectal balloons can be used to reduce treatment-related toxicity by sparing the posterior wall of the rectum $[7,8]$ and to restrict prostate motion by minimizing both inter- and intra-fractional variations in rectal position. In our previous report, a customized rectal balloon showed reliable positional reproducibility [9].

We have conducted hypofractionated IMRT (Hypo-IMRT, $70 \mathrm{~Gy}$ in $2.5 \mathrm{~Gy}$ fractions) with a customized rectal balloon since 2004. In this study, we aimed to analyze the treatment outcome and long-term toxicity of this protocol. As a reference, the results of this treatment were compared with those of 70.2 Gy delivered via conventionally fractionated 3-dimensional conformal radiotherapy (CF-3DRT).

\section{Materials and Methods}

\section{Patients}

Between January 2004 and December 2011, 86 patients with stages T1-T3b, NO-N1 localized prostate cancer were treated with definitive radiotherapy to a total dose of $70 \mathrm{~Gy}$ using a customized rectal balloon. Thirty patients received HypoIMRT, and 56 patients received CF-3DRT. All patients had pathologically confirmed prostate adenocarcinoma, acinar type. Patients who had previously received radiotherapy to the pelvis were excluded. On the basis of the Memorial Sloan Kettering Cancer Center risk grouping criteria, patients were divided into 3 prognostic groups as follows: low risk (initial PSA $\leq 10.0 \mathrm{ng} / \mathrm{mL}$; Gleason score [GS], 2-6; Stage T1a-T2b), intermediate risk (initial PSA $>10.0 \mathrm{ng} / \mathrm{mL}$ or GS $\geq 7$ or Stage $\geq T 2 \mathrm{c}$ ), and high risk (2 or 3 of the intermediate risk factors) [10]. The pretreatment evaluation consisted of a complete medical history, a digital rectal exam, a whole-body bone scan, a computed tomography (CT) scan of the abdomen and pelvis, magnetic resonance imaging of the prostate, and laboratory tests including measurement of serum prostate-specific antigen (PSA) levels.

\section{Radiotherapy}

All patients were instructed to empty their rectum by selfadministered enemas. Patients underwent CT simulation in the supine position after immobilization with a vacuum body fixation device. In order to minimize the effects of bladder motion, the bladder was filled for a specific period of time within a comfortable range for the patient.

Target volumes were defined as follows: gross tumor volume (GTV) included the prostate gland and any extraprostatic gross tumor and involved lymph nodes. Clinical target volume 1 (CTV1) included periprostatic fat tissue and proximal seminal vesicles, and CTV2 included the distal seminal vesicles and pelvic lymph nodes. Planning target volume 1 (PTV1) was defined as GTV plus a 5-mm margin in all directions except posteriorly, which had only a 2-mm margin to minimize rectal mucosal exposure. PTV2 and PTV3 were defined as CTV1 and CTV2, respectively, plus a 3-mm margin.

Total doses of 70 Gy (2.5 Gy/fraction), 60.2 Gy (2.15 Gy/ fraction), and 50.4 Gy (1.8 Gy/fraction) in 28 fractions were the prescribed dose-volumes for PTV1, PTV2, and PTV3, respectively. Assuming an $\alpha / \beta$ ratio of $3 \mathrm{~Gy}$, the doses delivered for PTV1, PTV2, and PTV3 via Hypo-IMRT equate to a $1.8 \mathrm{~Gy}$ equivalent dose of $81 \mathrm{~Gy}, 64.8 \mathrm{~Gy}$, and $50.4 \mathrm{~Gy}$, respectively. We applied rectal balloons to Hypo-IMRT patients at the beginning since the hypofractionated regimen reduces the treatment period by more than 2 weeks. Elective pelvic lymph node irradiation was performed only when the possibility of pelvic lymph node metastasis was greater than $30 \%$, as predicted by the Roach score for lymph node metastasis (RSLN)-based on the equation, 2/3 PSA + (GS - 6) $\times 10$ [11]. In patients with an RSLN of $30 \%$ or less, the pelvic lymph node chain was excluded from the PTV3. Eight patients underwent linacbased IMRT, and the others underwent helical tomotherapy. Linac-based IMRT was planned using the Corvus (Nomos Corp., Pittsburg, PA, USA) system, and helical tomotherapy using the Hi-Art (TomoTherapy Inc., Madison, WI, USA) planning station. The criterion for planning the PTV was that at least 95\% of it should be covered by $95 \%$ of the prescribed dose. The dosevolume constraints for normal tissues in the rectum were as follows: less than $60 \%$ of the volume received $30 \mathrm{~Gy}$, less than 15\% received $60 \mathrm{~Gy}$, and less than 5\% received $70 \mathrm{~Gy}$. For normal tissues in the bladder, less than $50 \%$ of the volume received 20 Gy. Less than 10\% of the femoral heads received $40 \mathrm{~Gy}$.

CF-3DRT was planned using the ADAC Pinnacle ${ }^{3}$ system, and the fraction dose was $1.8 \mathrm{~Gy}$. Distal seminal vesicles and/or pelvic lymph nodes were excluded through the first cone down at 45.0-50.4 Gy, and proximal seminal vesicle and periprostatic fat were excluded through the second cone down at $59.4 \mathrm{~Gy}$. 
Table 1. Rectal and bladder dose statistics according to the radiotherapy technique and field

\begin{tabular}{|c|c|c|c|c|}
\hline & \multicolumn{2}{|c|}{ Hypo-IMRT } & \multicolumn{2}{|c|}{ CF-3DRT } \\
\hline & Prostate (\%) & Prostate + WP (\%) & Prostate (\%) & Prostate + WP (\%) \\
\hline \multicolumn{5}{|l|}{ Rectum } \\
\hline$V_{5}$ & $97.4(93.0-100)$ & $99.1(95.0-100)$ & 100 & 100 \\
\hline$V_{10}$ & $94.2(89.0-100)$ & $98.1(92.5-100)$ & 100 & 100 \\
\hline$V_{55}$ & $13.5(0.5-21.0)$ & $8.2(1.0-18.0)$ & $44.0(34.0-68.2)$ & $49.0(34.8-66.2)$ \\
\hline$V_{60}$ & $10.4(0-15.0)$ & $5.1(0-14.0)$ & $34.1(28.0-46.0)$ & $35.0(24.3-57.8)$ \\
\hline$V_{65}$ & $6.3(0-10.0)$ & $3.1(0-10.0)$ & $19.5(15.0-35.0)$ & $25.0(17.0-35.7)$ \\
\hline$V_{70}$ & $2.5(0-5.0)$ & $0.8(0-2.5)$ & $14.8(8.2-22.0)$ & $18.9(10.8-23.6)$ \\
\hline$D_{\text {mean }}(G y)$ & $34.9(28.3-43.5)$ & $37.6(32.9-42.2)$ & $41.9(38.6-53.1)$ & $54.2(38.0-60.1)$ \\
\hline$D_{\max }(G y)$ & $73.1(71.1-78.7)$ & 73.2 (67.8-78.0) & $72.0(70.2-76.3)$ & $72.3(69.8-78.3)$ \\
\hline \multicolumn{5}{|l|}{ Bladder } \\
\hline$V_{5}$ & $99.6(98.0-100)$ & 100 & 100 & 100 \\
\hline$V_{10}$ & $97.0(90.0-100)$ & 100 & 100 & 100 \\
\hline$V_{55}$ & $6.0(1.0-17.0)$ & $10.5(2.0-20.0)$ & $37.4(30.5-50.2)$ & 41.5 (36.0-56.2) \\
\hline$V_{60}$ & $3.3(0-10.0)$ & $6.8(1.0-15.0)$ & $27.0(28.0-46.0)$ & $32.0(24.3-53.8)$ \\
\hline$V_{65}$ & $1.5(0-4.0)$ & $3.6(1.0-7.0)$ & $20.3(15.0-28.0)$ & $21.0(16.0-32.7)$ \\
\hline$V_{70}$ & $0.6(0-1.5)$ & $1.5(0-4.0)$ & $11.8(8.2-16.0)$ & $12.4(11.3-22.7)$ \\
\hline $\mathrm{D}_{\text {mean }}(\mathrm{Gy})$ & $36.4(31.7-43.1)$ & $35.9(16.0-46.4)$ & $44.7(38.6-54.2)$ & $46.3(39.1-58.3)$ \\
\hline $\mathrm{D}_{\max }(\mathrm{Gy})$ & $67.6(56.1-79.7)$ & $72.0(58.6-79.9)$ & $63.0(60.2-76.3)$ & $72.0(60.8-74.3)$ \\
\hline
\end{tabular}

Values are presented as mean (range).

CF-3DRT, conventionally fractionated 3-dimensional conformal radiotherapy; Hypo-IMRT, hypofractionated intensity-modulated radiotherapy; WP, whole pelvis; $V_{x}=$ organ percent volume receiving $x \mathrm{~Gy} ; D_{\text {meanı }}$ mean dose of the organ; $D_{\text {max }}$ maximum dose of the organ.

We prescribed up to 70.2 Gy for the GTV isocenter. In CF-3DRT group, the rectal balloon was not applied until the first cone down because it was thought that 39 fractions would be a burden to both the radiation therapist and the patient.

The dose statistics of the rectum and bladder are summarized according to the radiotherapy technique and field in Table 1.

\section{Androgen deprivation therapy}

Androgen deprivation therapy (ADT) was prescribed at the discretion of the treating urologist. In general, low- and intermediate-risk patients received a 4- to 6-month course of ADT, and high-risk patients received a 6-month to 2-year course of ADT before radiation therapy was considered. The median duration of ADT was 5.5 months (range, 0.73 to 93 months); it was 5.8 months (range, 0.73 to 36.4 months) for the Hypo-IMRT group and 5.3 months (range, 0.73 to 93 months) for the CF-3DRT group ( $p=0.938)$.

\section{Follow-ups and toxicities}

All end points were calculated from the completion of the radiation treatment. Biochemical failure (BCF) was defined using the Phoenix consensus of the nadir of PSA concentration plus $2 \mathrm{ng} / \mathrm{mL}$. Death was considered as cancer-specific if there was clear evidence that it occurred secondary to prostate cancer or if the patient had metastatic disease with an elevated PSA level at the time of death.

Acute and late toxicities were assessed using the Radiation Therapy Oncology Group morbidity criteria. Acute toxicity was defined as any new event occurring during radiotherapy or within 3 months after radiotherapy, and late toxicities as any event persisting for more than 3 months or occurring more than 3 months after radiotherapy. Patients were seen 3 and 6 months after completion of radiotherapy and every 6 months thereafter.

\section{Statistical analysis}

Kaplan-Meier curves for biochemical relapse-free survival (BRFS) and overall survival were generated, and survival comparisons were made using the log-rank test. Clinical characteristics and toxicity grading among the treatment groups were compared using the chi-square test or Fisher exact test for categorical variables. Multivariable analysis was performed via a proportional hazards analysis, using prespecified categorical explanatory variables (e.g., clinical 
Table 2. Characteristics and treatments of the patients

\begin{tabular}{|c|c|c|c|c|}
\hline & All $(n=86)$ & Нypo-IMRT $(n=30)$ & CF-3DRT $(n=56)$ & $p$-value \\
\hline Age (yr) & 71.5 (55-88) & 70.8 (60-79) & $72(55-88)$ & 0.938 \\
\hline cT stage & & & & 0.526 \\
\hline T1-T2a & $26(30.2)$ & $9(30.0)$ & $17(30.4)$ & \\
\hline T2b-T2c & $17(19.8)$ & 4 (13.3) & $13(23.2)$ & \\
\hline ТЗа-ТЗь & $43(50.0)$ & $17(56.6)$ & $26(46.4)$ & \\
\hline Gleason score & & & & 0.754 \\
\hline $5-6$ & $25(29.1)$ & 10 (33.3) & $15(26.8)$ & \\
\hline 7 & $30(34.9)$ & $11(36.7)$ & 19 (33.9) & \\
\hline 8-10 & $31(36.0)$ & $9(30)$ & $22(39.3)$ & \\
\hline cN stage & & & & 0.534 \\
\hline 0 & $79(91.9)$ & 28 (93.3) & $51(91.1)$ & \\
\hline 1 & $7(8.1)$ & $2(6.7)$ & $5(8.9)$ & \\
\hline Initial PSA & & & & 0.788 \\
\hline$\leq 10$ & $30(34.9)$ & $12(40.0)$ & $18(21.1)$ & \\
\hline$>10, \leq 20$ & $21(24.4)$ & $6(20.0)$ & $15(26.8)$ & \\
\hline$>20$ & $35(40.7)$ & $12(40.0)$ & $23(41.1)$ & \\
\hline Risk group & & & & 0.779 \\
\hline Low & $11(12.8)$ & $5(16.6)$ & $6(10.7)$ & \\
\hline Intermediate & $18(20.9)$ & $5(16.6)$ & $13(23.2)$ & \\
\hline High & $57(66.3)$ & $20(66.7)$ & $37(66.1)$ & \\
\hline RSLN (\%), 30 & & & & 0.634 \\
\hline$>30$ & $47(54.7)$ & $15(50.0)$ & $32(57.1)$ & \\
\hline$\leq 30$ & $39(45.3)$ & $15(50.0)$ & $24(42.9)$ & \\
\hline RSLN (\%), 15 & & & & 0.594 \\
\hline$>15$ & $58(67.4)$ & $20(66.7)$ & $38(67.8)$ & \\
\hline$\leq 15$ & $28(32.6)$ & 10 (33.3) & $18(32.2)$ & \\
\hline RT field & & & & 0.634 \\
\hline Prostate & $47(54.7)$ & $15(50.0)$ & $32(57.1)$ & \\
\hline WP & $39(45.3)$ & $15(50.0)$ & $24(42.9)$ & \\
\hline ADT & & & & 0.657 \\
\hline No & $39(45.3)$ & 13 (43.3) & $26(46.4)$ & \\
\hline Yes & $47(54.7)$ & $17(56.7)$ & $30(53.6)$ & \\
\hline Duration (mo) & $5.5(0.73-93.0)$ & $5.8(0.73-36.4)$ & $5.3(0.73-93.0)$ & 0.938 \\
\hline
\end{tabular}

Values are presented as median (range) or number (\%).

ADT, androgen-deprivation therapy; CF-3DRT, conventionally fractionated 3-dimensional conformal radiotherapy; CN, clinical nodal; cT, clinical tumor; Hypo-IMRT, hypofractionated intensity-modulated radiotherapy; PSA, prostate-specific antigen; RSLN, Roach score for prediction of lymph node metastasis; RT, radiotherapy; WP, whole pelvis.

stage, GS, PSA, and radiation dose). Variables associated with late toxicity were evaluated using logistic regression. A p-value $<0.05$ was considered statistically significant. Statistical analysis was performed using SPSS ver. 20.0.0 software (SPSS Inc., Chicago, IL, USA).

\section{Results}

\section{Patients and treatments}

Table 2 lists the characteristics and treatments of the patients.
No significant differences were found in the age, clinical T stage, GS, nodal stage, initial PSA level, risk group, RSLN, radiotherapy field, or use of ADT between the Hypo-IMRT and CF-3DRT groups. The median PSA level at diagnosis was $14.08 \mathrm{ng} / \mathrm{mL}$ (range, 3.6 to $180.2 \mathrm{ng} / \mathrm{mL}$ ). The percentages of patients in the low-, intermediate-, and high-risk groups were $12.8 \%, 20.9 \%$, and $66.3 \%$, respectively. RSLNs were divided into 2 categories based on cutoff values of 15\%, which is traditionally used, and 30\%. ADT was administered to 47 patients, 17 (56.7\%) in the Hypo-IMRT group and 30 (53.6\%) 
A

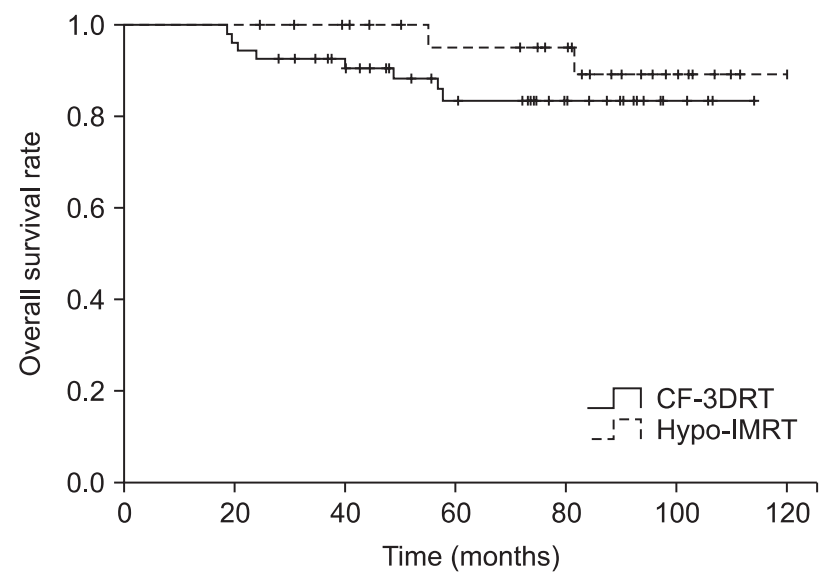

B

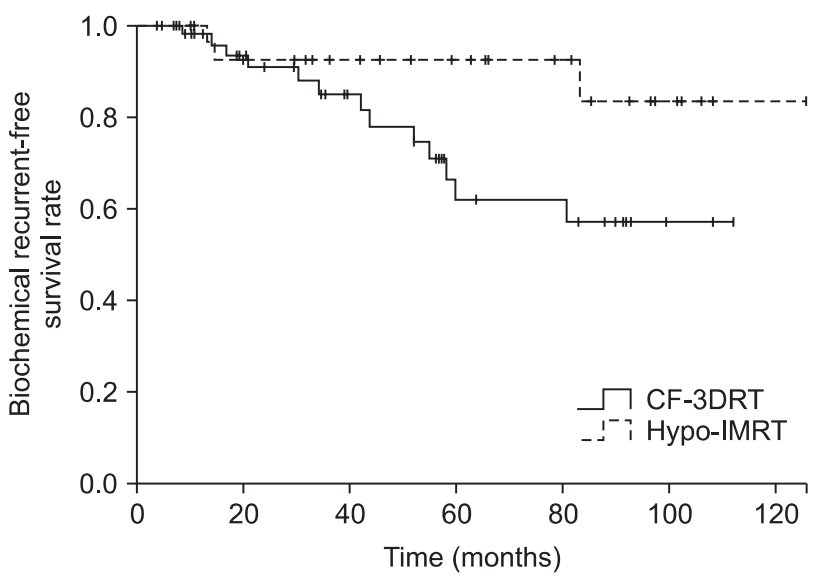

Fig. 1. Overall survival (A) and biochemical recurrence-free survival (B) for conventionally fractionated 3-dimensional conformal radiotherapy (CF-3DRT) and hypofractionated intensity-modulated radiotherapy (Hypo-IMRT).

in the CF-3DRT group. The Hypo-IMRT group included 2 lowrisk (40\% of all low-risk patients), 2 intermediate-risk (40\% of all intermediate-risk patients), and 13 high-risk (65\% of all high-risk patients) ADT recipients. The CF-3DRT group included 1 low-risk (16.7\%), 4 intermediate-risk (30.8\%), and 25 highrisk (67.6\%) ADT recipients.

\section{Biochemical control and survival}

The median follow-up time, defined as the interval from the completion of radiotherapy to the last PSA measurement, was 74.4 months (range, 18.8 to 125.9 months); the median time was 73.4 months (range, 18.8 to 114.07 months) for the HypoIMRT group and 78.6 months (range, 20 to 125.9 months) for the CF-3DRT group. The actuarial BRFS rate for all patients at 5 years was 75.8\%; it was 92.9\% for the Hypo-IMRT group and $64.1 \%$ for the CF-3DRT group (log-rank, $p<0.046$ ) (Fig. 1). The 5-year BRFS rates for low-, intermediate-, and high-risk patients were $100 \%, 100 \%$, and $88.5 \%$, respectively, for the Hypo-IMRT group and 80\%, 77.8\%, and 63.6\%, respectively, for the CF-3DRT group (Fig. 2).

A univariate analysis demonstrated that administration of ADT, an RSLN over 30, and EQD1.8 $\mathrm{Gy}_{3}$ were associated with biochemical outcome (Table 3). EQD1.8 $\mathrm{Gy}_{3}$ is defined as the 1.8 Gy-equivalent dose, assuming an $\alpha / \beta$ ratio of $3 \mathrm{~Gy}$. Men receiving ADT had an overall BRFS rate of $78.6 \%$ compared with $55.4 \%$ for men who did not receive ADT $(p=0.015)$. The 5-year BRFS rates were $89.4 \%$ and $72.3 \%$ for ADT recipients in the hypo-IMRT group and CF-3DRT group, respectively. Men with an RSLN over 30 had a 5-year BRFS rate of $67.5 \%$ compared with $88.2 \%$ for men with an RSLN less than 30 ( $p$
$=0.019)$. In a multivariable analysis, administration of ADT (hazard ratio, 5.705; $p=0.002$ ) and an RSLN over 30 (hazard ratio, $0.185 ; p=0.005)$ were associated with biochemical outcome, whereas EQD1.8 $\mathrm{Gy}_{3}$ was not.

The overall survival rate was $87.9 \%$ at 5 years. No prostate cancer-specific deaths were observed in any of the risk groups. During the follow-up period, 12 patients died of pneumonia, heart failure, or accidental events.

\section{Analysis of biochemical failures}

Three (10\%) of the 30 patients in the Hypo-IMRT group experienced BCF. All 3 were high-risk patients. Two developed BCF 15 and 16 months after radiotherapy as their sole treatment; both received hormone therapy after $\mathrm{BCF}$, one of whom developed sternal metastasis 85 months after treatment. The third patient experienced BCF 83 months after an initial 5-month hormone treatment and subsequent radiotherapy. In the CF-3DRT group, 13 patients (23\%) experienced BCF with a median time to failure of 42 months (range, 9 to 80 months). These included 1, 3, and 9 patients in the low-, intermediate-, and high-risk groups, respectively. The low- to intermediaterisk patients received radiotherapy alone before BCF and hormone therapy after BCF. The high-risk patients experienced BCF at a median time of 30 months (range, 9 to 80 months). Among them, 4 had initially received radiotherapy alone, and 5 had received combined hormone therapy and radiotherapy.

\section{Late toxicity}

The late toxicities of all patients are summarized in Table 4 . The 5-year actuarial grade 2 or higher late gastrointestinal (GI) 
A

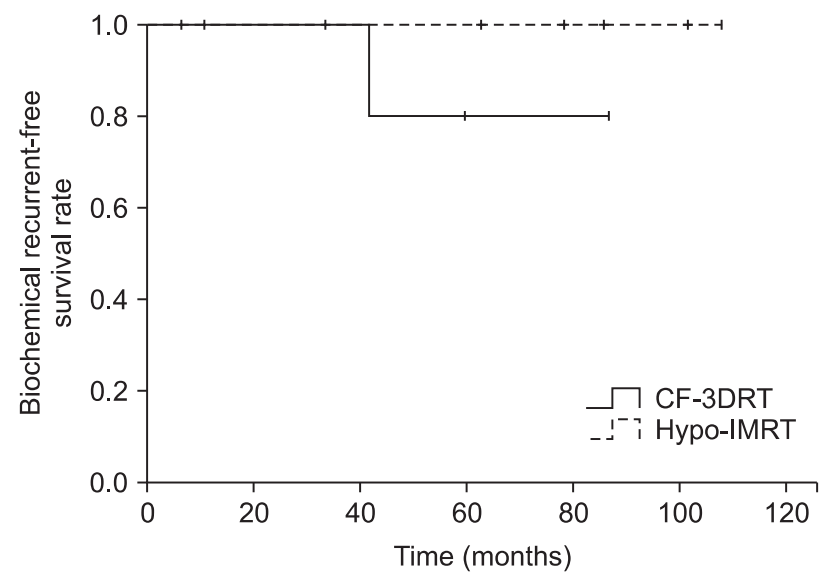

C

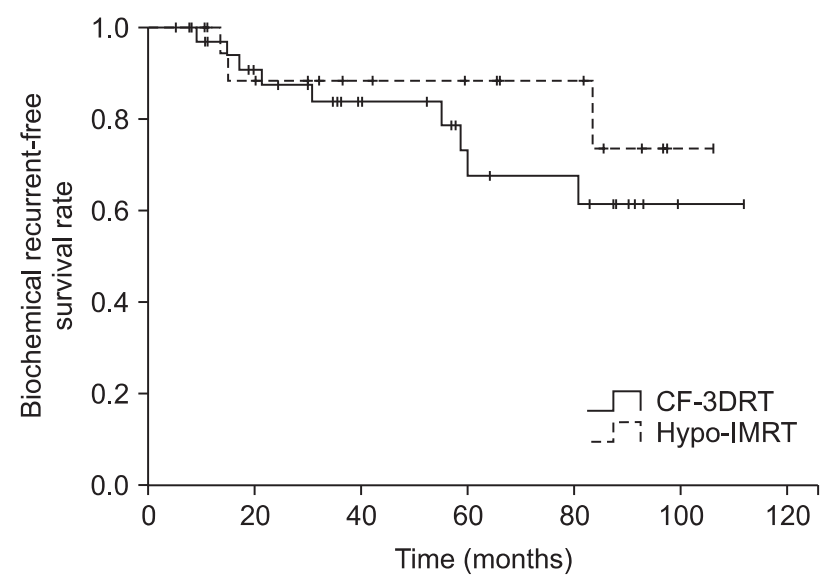

B

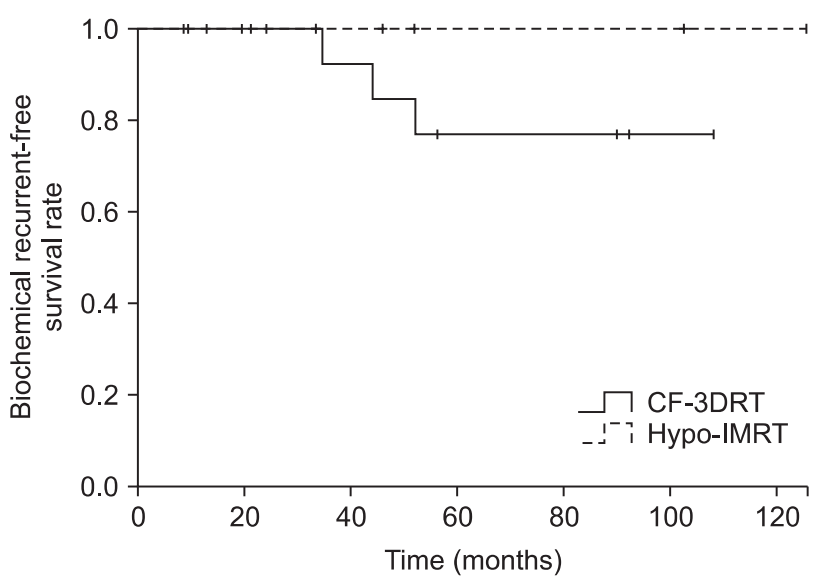

Fig. 2. Biochemical recurrence-free survival for conventionally fractionated 3-dimensional conformal radiotherapy (CF-3DRT) and hypofractionated intensity-modulated radiotherapy (HypoIMRT) in the low-risk group (A), intermediate-risk group (B), and high-risk group (C).

Table 3. Univariate and multivariate analysis for biochemical relapse free survival influencing factors

\begin{tabular}{|c|c|c|c|c|}
\hline & \multirow{2}{*}{ Variable } & \multirow{2}{*}{$\begin{array}{c}\text { Univariate } \\
\text { p-value }\end{array}$} & \multicolumn{2}{|c|}{ Multivariate } \\
\hline & & & $\mathrm{HR}(95 \% \mathrm{Cl})$ & p-value \\
\hline Age (yr) & $<70$ vs. $\geq 70$ & 0.514 & - & - \\
\hline Risk group & Low vs. Intermediate vs. High & 0.818 & - & - \\
\hline Gleason score & $>7$ vs. 7 vs. $7<$ & 0.578 & - & - \\
\hline cT stage & T1-T2a vs. T2b-T2c vs. T3 & 0.242 & - & - \\
\hline ¡PSA (ng/mL) & $\leq 10$ vs. $>10$ and $\leq 20$ vs. $>20$ & 0.210 & - & - \\
\hline \multirow[t]{2}{*}{ RSLN (\%) } & $\leq 30$ vs. $>30$ & 0.019 & $0.185(0.058-0.593)$ & 0.005 \\
\hline & $\leq 15$ vs. $>15$ & 0.371 & - & - \\
\hline RT field & Whole pelvis vs. Prostate & 0.300 & - & - \\
\hline EQD1.8 $\mathrm{Gy}_{3}$ & 81 vs. 70.2 & 0.046 & $2.257(0.613-8.311)$ & 0.221 \\
\hline Use of ADT & Yes vs. No & 0.015 & 5.705 (1.880-17.315) & 0.002 \\
\hline
\end{tabular}

ADT, androgen deprivation therapy; cT, clinical tumor; EQD1.8 Gy3, 1.8 Gy equivalent dose, assuming an $\alpha / \beta$ ratio of 3 Gy; iPSA, initial prostate-specific antigen; RSLN, Roach score for prediction of lymph node metastasis; RT, radiotherapy; ADT, androgen deprivation therapy.

toxicity rate was 7\%. Three patients experienced late grade $3 \mathrm{GI}$ toxicity, 2 (3.6\%) in the CF-3DRT group and 1 (3.3\%) in the Hypo-IMRT group. These patients were treated by argon plasma coagulation and are now symptom-free. Two patients (2.3\%) experienced late genitourinary (GU) grade 2 toxicity, $1(1.8 \%)$ in the CF-3DRT group and $1(3.3 \%)$ in the Hypo- 
Table 4. Crude incidence of maximum late genitourinary and gastrointestinal morbidity between men who received Hypo-IMRT and CF-3DRT

\begin{tabular}{|c|c|c|c|c|c|c|}
\hline \multirow{2}{*}{ Toxicity } & \multicolumn{5}{|c|}{ RTOG toxicity grade } & \multirow{2}{*}{$p$-value } \\
\hline & 0 & 1 & 2 & 3 & 4 & \\
\hline Late GU & & & & & & 0.579 \\
\hline CF-3DRT & $49(87.5)$ & 8 (14.3) & $1(1.8)$ & 0 & 0 & \\
\hline Нypo-IMRT & 25 (83.3) & $4(13.3)$ & $1(3.3)$ & 0 & 0 & \\
\hline Late GI & & & & & & 0.653 \\
\hline CF-3DRT & 50 (89.3) & $2(3.6)$ & $2(3.6)$ & $2(3.6)$ & 0 & \\
\hline Hypo-IMRT & $27(90.0)$ & $1(3.3)$ & $1(3.3)$ & $1(3.3)$ & 0 & \\
\hline
\end{tabular}

Values are presented as number (\%).

Hypo-IMRT, hypofractionated intensity-modulated radiotherapy; CF-3DRT, conventionally fractionated radiotherapy; RTOG, Radiation Therapy Oncology Group; GU, genitourinary; GI, gastrointestinal.

${ }^{\text {a) }}$ Toxicity that developed $\geq 90$ days after completion of radiotherapy.

Table 5. Analysis of variables affecting late genitourinary and gastrointestinal toxicity

\begin{tabular}{|c|c|c|c|c|c|}
\hline & \multirow{2}{*}{ Variable } & \multicolumn{2}{|c|}{ Late $\mathrm{GU} \geq$ grade 2} & \multicolumn{2}{|c|}{ Late $\mathrm{Gl} \geq$ grade 2} \\
\hline & & OR $(95 \% \mathrm{Cl})$ & p-value & OR (95\% Cl) & $p$-value \\
\hline Age (yr) & $\geq 70$ vs. $<70$ & - & 0.998 & $2.413(0.238-19.332)$ & 0.497 \\
\hline DM & Yes vs. no & - & 0.998 & - & 0.998 \\
\hline Anticoagulant use & Yes vs. no & - & 0.998 & $0.800(0.087-7.335)$ & 0.844 \\
\hline Hemorrhoid & Yes vs. no & - & 0.999 & $5.133(0.449-58.732)$ & 0.188 \\
\hline $\mathrm{BPH}$ & Yes vs. no & - & 0.997 & - & - \\
\hline ADT & Yes vs. no & - & 0.998 & $1.721(0.298-9.937)$ & 0.544 \\
\hline Acute GU toxicity & Yes vs. no & $1.758(0.251-12.330)$ & 0.570 & - & - \\
\hline Acute GI toxicity & Yes vs. no & - & - & 2.547 (0.983-6.602) & 0.054 \\
\hline \multirow[t]{2}{*}{ Risk group } & Low vs. intermediate & - & 0.999 & $2.750(0.227-33.276)$ & 0.426 \\
\hline & Intermediate vs. high & $3.294(0.195-55.511)$ & 0.408 & $5.500(0.841-35.975)$ & 0.075 \\
\hline EOD1.8 $\mathrm{Gy}_{3}$ & 81 vs. 70.2 & $0.527(0.032-8.741)$ & 0.655 & $1.077(0.186-6.250)$ & 0.934 \\
\hline Rectal balloon & First vs. at cone down & 0.527 (0.032-8.741) & 0.655 & $1.077(0.186-6.250)$ & 0.934 \\
\hline RT field & Prostate vs. whole pelvis & $0.787(0.048-13.012)$ & 0.867 & $0.787(0.048-13.012)$ & 0.867 \\
\hline
\end{tabular}

$\mathrm{ADT}$, androgen deprivation therapy; $\mathrm{BPH}$, benign prostate hyperplasia; $\mathrm{Cl}$, confidence interval; $\mathrm{DM}$, diabetes mellitus; EQD1.8 Gy ${ }_{3}, 1.8 \mathrm{~Gy}$ equivalent dose, assuming an $\alpha / \beta$ of $3 \mathrm{~Gy}$; GI, gastrointestinal; $\mathrm{GU}$, genitourinary; OR, odds ratio; RT, radiotherapy.

IMRT group. No grade 3 or higher GU complications were encountered. We performed a binary-logistic regression to identify the variables causing late GU and GI toxicity (Table 5). Patients who had experienced acute GI toxicity above grade 2 during radiotherapy had a tendency of developing late grade $2 \mathrm{Gl}$ toxicity or above (odds ratio, 2.547; 95\% confidence interval, 0.983-6.602; $p=0.054)$.

\section{Discussion and Conclusion}

Our results revealed that an excellent 5-year BRFS rate of 92.9\% could be achieved when 70 Gy were delivered in 2.5Gy daily fractions via Hypo-IMRT for localized prostate cancer.
Furthermore, although its biologically effective dose was higher than that of CF-3DRT, Hypo-IMRT resulted in similar or less $\mathrm{GI}$ and $\mathrm{GU}$ toxicities in the normal tissues. We attribute this enhancement of the therapeutic ratio to the improved isodose distribution of IMRT, the hypofractionation scheme, and the rectal immobilization and poseterior wall sparing achieved by a customized rectal balloon. To our knowledge, this is the first report of definitive radiotherapy performed together with a rectal balloon, using a dose escalated hypofractionation scheme in the management of localized prostate cancer in Korea.

As a result of the several randomized control trials [12-14] of prostate cancer radiotherapy, we can expect that the dose- 


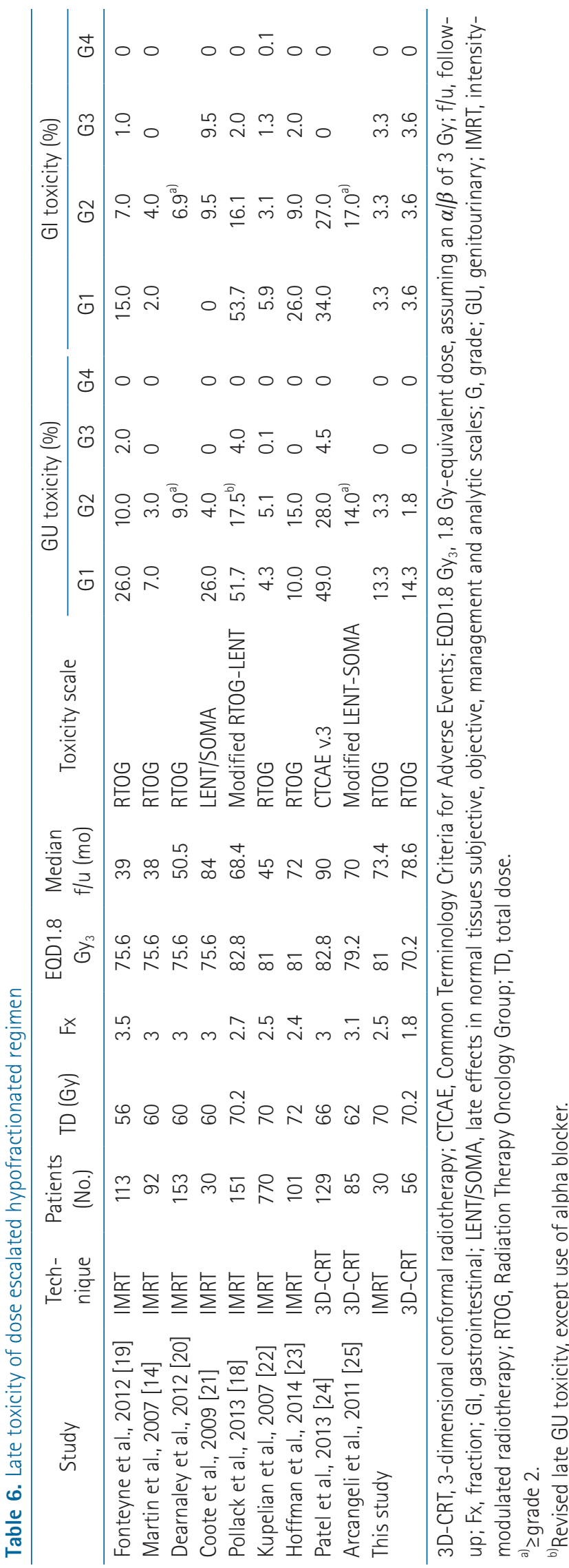

escalations of higher than $10 \mathrm{~Gy}$ in conventional fractionation might increase BRFS of prostate cancer patients. However, treatments using conventional fractionation exceeding 75-80 Gy require a long treatment period (7-8 weeks); and Thames et al. [12] reported that this prolonged overall treatment time reduced the BRFS. On the other hand, prostate cancer is characterized by a low $\alpha / \beta$ ratio and slow cell kinetics $[4,5]$, whereas the rectum, which is adjacent to the prostate, has a relatively high $\alpha / \beta$ ratio [6]; therefore, hypofractionation would be beneficial. As such, if the biological effective dose, which is similar to the conventionally escalated total dose, is applied during a hypofractionated treatment schedule, we can expect a high BRFS along with a reduced treatment period. However, as no firm optimal dose scheme has been specified, various dose-escalated hypofractionation schemes have been attempted by different institutions [13-17]. Since the early 2000s, patients undergoing IMRT at our institute have received radiation treatments comprising a total dose of $70 \mathrm{~Gy}$ in 2.5-Gy fractions, or dose-escalated hypofractionated regimens.

Although opinions vary with respect to the correct $\alpha / \beta$ ratio for prostate cancer, Oliveira et al. [4] estimated this value as approximately $2.7 \mathrm{~Gy}$ based on their literature review. In our study, the $\alpha / \beta$ ratio was set to $3 \mathrm{~Gy}$ in order to convert the biological equivalent doses for comparisons of treatment outcomes and toxicities. The regimen comprising $70 \mathrm{~Gy}$ in 2.5-Gy fractions used in our study was delivered at an equivalent dose of $1.8 \mathrm{~Gy}$ per fraction (EOD1.8) of $81 \mathrm{~Gy}_{3}$. In other studies in which the total biological equivalent doses also exceeded 80 Gy EQD $1.8[13-15,17,18]$, the BRFS rates for low-, intermediate-, and high-risk patients ranged between 94\%-98\%, 83\%-100\%, and 71\%-85\%, respectively. Most of these studies reported better BRFS rates relative to the other arms, in which patients received doses of approximately $70 \mathrm{~Gy}$, and our study also reconfirmed that the Hypo-IMRT group experienced better results than the CF-3DRT group, which was prescribed a dose of only $70.2 \mathrm{~Gy}$. Specifically, the 5-year low-, intermediate-, and high-risk BRFS rates were $100 \%, 100 \%$, and $88.5 \%$, respectively in this study. Based on the treatment results obtained according to the institutional protocols investigated to date, the present scheme appears sufficient for low- to intermediate-risk patients, while some limitations still exist for high-risk patients. Therefore, to reduce these failures, further dose-escalation trials using externalbeam radiotherapy dose increases or combination therapy including brachytherapy should be conducted.

The late toxicities experienced in the dose-escalated hypo- 
fractionated trials at various institutes $[14,18-25]$ are listed in Table 6 . Although toxicity comparisons should be interpreted carefully according to the selected scale [26], studies using the Radiation Therapy Oncology Group scale reported that among cases of GU toxicity, approximately $3.0 \%-15.0 \%$ of patients developed grade 2 or higher toxicities, including a few cases of grade 3 toxicity; in cases of GI toxicity, approximately 4.0\%-11.0\% of patients developed grade 2 or higher side effects. In studies involving IMRT, the late toxicity levels have been reported as acceptable. Accordingly, in the present study, although 1 patient developed a grade $3 \mathrm{GI}$ toxicity in the Hypo-IMRT group, the percentage of patients who developed grade 2 or higher toxicities was low. This may be attributed to the use rectal balloons in our institute. This rectal balloon, the effectiveness of which has been previously reported by Teh et al. [8] and Smeenk et al. [27], acts as a fiducial marker in image-guided radiotherapy and can fixate the prostate to render it immovable. Furthermore, the balloon excludes the posterior rectal wall from the radiation treatment field, thereby exposing it to a minimal radiation dose and reducing the rectal toxicity. The symmetric rectal balloon designed at our institute can stably fixate the prostate and also features superior positional reproducibility in the rectum [9]. In an actual treatment situation, because the rectal balloon must be inserted for each treatment, cooperation between the patient and the radiotherapist is essential; however, with prolonged treatment, the patient's compliance may decrease. By reducing the treatment period, hypofractionated radiotherapy is considered to increase the patient's acceptance of the rectal balloon.

Currently, there is much controversy regarding whether elective pelvic irradiation used during prostate cancer treatment can reduce pelvic nodal recurrences and whether this technique will positively influence the treatment outcome. Usually, an RSLN of $15 \%[11,28,29]$ is recommended as the indication for performing elective pelvic irradiation. However, negative elective nodal irradiation results have been recently reported in some randomized trials [30-32]. At our institute, we perform elective nodal irradiation only when the RSLN exceeds 30\%, which is a much stricter cutoff than 15\%. As a result, no nodal recurrence occurred in patients who did not undergo pelvic nodal irradiation; and, interestingly, an RSLN of 30\% was found to be the main prognostic factor for BRFS. Mantini et al. [33] reported that patients with high-risk nodal involvement ( $>30 \%$ RSLN) who underwent whole pelvis radiotherapy exhibited statistically significant improvements in disease-free survival relative to those receiving prostate-only radiotherapy.

Although this study was conducted according to a consistent dose scheme and target definition, it has some limitations, owing to the relatively small number of patients compared with those included in Western reports, as well as the retrospective nature of the study.

In conclusion, the results of the present study revealed an increase in the BRFS resulting from the use of a dose-escalated hypofractionation dose scheme; along with the maintenance of a low toxicity rate, owing to the use of a customized rectal balloon together with IMRT.

\section{Conflict of Interest}

No potential conflict of interest relevant to this article was reported.

\section{Acknowledgments}

The authors thank HY Kim (Biostatistics Collaboration Unit, Yonsei University College of Medicine, Seoul, Korea) for statistical analysis consultation.

\section{References}

1. Symon Z, Griffith KA, McLaughlin PW, Sullivan M, Sandler HM. Dose escalation for localized prostate cancer: substantial benefit observed with 3D conformal therapy. Int J Radiat Oncol Biol Phys 2003;57:384-90.

2. Pollack $A$, Hanlon $A$, Horwitz EM, Feigenberg $S$, Uzzo RG, Price RA. Radiation therapy dose escalation for prostate cancer: a rationale for IMRT. World J Urol 2003;21:200-8.

3. Zelefsky MJ, Fuks $Z$, Hunt $M$, et al. High dose radiation delivered by intensity modulated conformal radiotherapy improves the outcome of localized prostate cancer. J Urol 2001;166:876-81.

4. Oliveira SM, Teixeira NJ, Fernandes L. What do we know about the $\alpha / \beta$ for prostate cancer? Med Phys 2012;39:3189-201.

5. Daşu A. Is the alpha/beta value for prostate tumours low enough to be safely used in clinical trials? Clin Oncol (R Coll Radiol) 2007;19:289-301.

6. Brenner DJ. Fractionation and late rectal toxicity. Int J Radiat Oncol Biol Phys 2004;60:1013-5.

7. Wachter $S$, Gerstner N, Dorner D, et al. The influence of a rectal balloon tube as internal immobilization device on variations of volumes and dose-volume histograms during 
treatment course of conformal radiotherapy for prostate cancer. Int J Radiat Oncol Biol Phys 2002;52:91-100.

8. Teh BS, McGary JE, Dong L, et al. The use of rectal balloon during the delivery of intensity modulated radiotherapy (IMRT) for prostate cancer: more than just a prostate gland immobilization device? Cancer J 2002;8:476-83.

9. Cho JH, Lee CG, Kang DR, et al. Positional reproducibility and effects of a rectal balloon in prostate cancer radiotherapy. J Korean Med Sci 2009;24:894-903.

10. Sylvester JE, Blasko JC, Grimm PD, Meier R, Malmgren JA. Tenyear biochemical relapse-free survival after external beam radiation and brachytherapy for localized prostate cancer: the Seattle experience. Int J Radiat Oncol Biol Phys 2003;57:94452.

11. Roach M 3rd, Marquez C, Yuo HS, et al. Predicting the risk of lymph node involvement using the pre-treatment prostate specific antigen and Gleason score in men with clinically localized prostate cancer. Int J Radiat Oncol Biol Phys 1994;28:33-7.

12. Thames HD, Kuban D, Levy LB, et al. The role of overall treatment time in the outcome of radiotherapy of prostate cancer: an analysis of biochemical failure in 4839 men treated between 1987 and 1995. Radiother Oncol 2010;96:6-12.

13. Kupelian PA, Thakkar W, Khuntia D, Reddy CA, Klein EA, Mahadevan A. Hypofractionated intensity-modulated radiotherapy (70 Gy at 2.5 Gy per fraction) for localized prostate cancer: long-term outcomes. Int J Radiat Oncol Biol Phys 2005;63:1463-8.

14. Martin JM, Rosewall T, Bayley A, et al. Phase II trial of hypofractionated image-guided intensity-modulated radiotherapy for localized prostate adenocarcinoma. Int J Radiat Oncol Biol Phys 2007;69:1084-9.

15. Faria S, Dal Pra A, Cury F, et al. Treating intermediate-risk prostate cancer with hypofractionated external beam radiotherapy alone. Radiother Oncol 2011;101:486-9.

16. Leborgne F, Fowler J, Leborgne JH, Mezzera J. Later outcomes and alpha/beta estimate from hypofractionated conformal three-dimensional radiotherapy versus standard fractionation for localized prostate cancer. Int J Radiat Oncol Biol Phys 2012;82:1200-7.

17. Arcangeli S, Strigari L, Gomellini S, et al. Updated results and patterns of failure in a randomized hypofractionation trial for high-risk prostate cancer. Int J Radiat Oncol Biol Phys 2012;84:1172-8.

18. Pollack A, Walker G, Horwitz EM, et al. Randomized trial of hypofractionated external-beam radiotherapy for prostate cancer. J Clin Oncol 2013;31:3860-8.

19. Fonteyne V, Soete G, Arcangeli S, et al. Hypofractionated highdose radiation therapy for prostate cancer: long-term results of a multi-institutional phase II trial. Int J Radiat Oncol Biol
Phys 2012;84:e483-90.

20. Dearnaley D, Syndikus I, Sumo G, et al. Conventional versus hypofractionated high-dose intensity-modulated radiotherapy for prostate cancer: preliminary safety results from the CHHiP randomised controlled trial. Lancet Oncol 2012;13:43-54.

21. Coote JH, Wylie JP, Cowan RA, Logue JP, Swindell R, Livsey JE. Hypofractionated intensity-modulated radiotherapy for carcinoma of the prostate: analysis of toxicity. Int J Radiat Oncol Biol Phys 2009;74:1121-7.

22. Kupelian PA, Willoughby TR, Reddy CA, Klein EA, Mahadevan A. Hypofractionated intensity-modulated radiotherapy (70 Gy at 2.5 Gy per fraction) for localized prostate cancer: Cleveland Clinic experience. Int J Radiat Oncol Biol Phys 2007;68:142430.

23. Hoffman KE, Voong KR, Pugh TJ, et al. Risk of late toxicity in men receiving dose-escalated hypofractionated intensity modulated prostate radiation therapy: results from a randomized trial. Int J Radiat Oncol Biol Phys 2014;88:107484.

24. Patel N, Faria $S$, Cury $F$, et al. Hypofractionated radiation therapy (66 Gy in 22 fractions at 3 Gy per fraction) for favorable-risk prostate cancer: long-term outcomes. Int J Radiat Oncol Biol Phys 2013;86:534-9.

25. Arcangeli G, Fowler J, Gomellini S, et al. Acute and late toxicity in a randomized trial of conventional versus hypofractionated three-dimensional conformal radiotherapy for prostate cancer. Int J Radiat Oncol Biol Phys 2011;79:1013-21.

26. Lund JA, Kaasa $S$, Wibe $A$, Widmark A, Fransson P. Late radiation effects to the rectum and anus after treatment for prostate cancer; validity of the LENT/SOMA score. Acta Oncol 2013;52:727-35.

27. Smeenk RJ, Louwe RJ, Langen KM, et al. An endorectal balloon reduces intrafraction prostate motion during radiotherapy. Int J Radiat Oncol Biol Phys 2012;83:661-9.

28. Aizer AA, Yu JB, McKeon AM, Decker RH, Colberg JW, Peschel RE. Whole pelvic radiotherapy versus prostate only radiotherapy in the management of locally advanced or aggressive prostate adenocarcinoma. Int J Radiat Oncol Biol Phys 2009;75:1344-9.

29. Seaward SA, Weinberg $V$, Lewis $P$, Leigh $B$, Phillips $T L$, Roach $M$ 3rd. Identification of a high-risk clinically localized prostate cancer subgroup receiving maximum benefit from wholepelvic irradiation. Cancer J Sci Am 1998;4:370-7.

30. Lawton CA, DeSilvio M, Roach M 3rd, et al. An update of the phase III trial comparing whole pelvic to prostate only radiotherapy and neoadjuvant to adjuvant total androgen suppression: updated analysis of RTOG 94-13, with emphasis on unexpected hormone/radiation interactions. Int J Radiat Oncol Biol Phys 2007;69:646-55.

31. Pommier $P$, Chabaud $S$, Lagrange $J$, et al. Is there a role for 
pelvic irradiation in localized prostate adenocarcinoma? Preliminary results of GETUG-01. J Clin Oncol 2007;25:536673.

32. Asbell SO, Martz KL, Shin $\mathrm{KH}$, et al. Impact of surgical staging in evaluating the radiotherapeutic outcome in RTOG \#77-06, a phase III study for T1BNOMO (A2) and T2NOMO (B) prostate carcinoma. Int J Radiat Oncol Biol Phys 1998;40:769-82.

33. Mantini G, Tagliaferri L, Mattiucci GC, et al. Effect of whole pelvic radiotherapy for patients with locally advanced prostate cancer treated with radiotherapy and long-term androgen deprivation therapy. Int J Radiat Oncol Biol Phys 2011;81:e721-6. 